\title{
Trace methane oxidation studied in several Euryarchaeota under diverse conditions
}

\author{
JAMES J. MORAN, ${ }^{1}$ CHRISTOPHER H. HOUSE, ${ }^{1,2}$ KATHERINE H. FREEMAN ${ }^{1}$ and JAMES \\ G. FERRY ${ }^{3}$ \\ ${ }^{1}$ Department of Geosciences and Penn State Astrobiology Research Center, Penn State University, 220 Deike Bldg., University Park, PA 16802, USA \\ 2 Corresponding author (chouse@geosc.psu.edu) \\ ${ }^{3}$ Department of Biochemistry and Molecular Biology and Penn State Astrobiology Research Center, Penn State University, 205 South Frear, \\ University Park, PA 16802, USA
}

Received June 16, 2004; accepted November 16, 2004; published online December 6, 2004

\begin{abstract}
Summary We used ${ }^{13} \mathrm{C}$-labeled methane to document the extent of trace methane oxidation by Archaeoglobus fulgidus, Archaeoglobus lithotrophicus, Archaeoglobus profundus, Methanobacterium thermoautotrophicum, Methanosarcina barkeri and Methanosarcina acetivorans. The results indicate trace methane oxidation during growth varied among different species and among methanogen cultures grown on different substrates. The extent of trace methane oxidation by $M b$. thermoautotrophicum $(0.05 \pm 0.04 \%, \pm 2$ standard deviations of the methane produced during growth) was less than that by $M$. barkeri $(0.15 \pm 0.04 \%)$, grown under similar conditions with $\mathrm{H}_{2}$ and $\mathrm{CO}_{2}$. Methanosarcina acetivorans oxidized more methane during growth on trimethylamine $(0.36 \pm 0.05 \%)$ than during growth on methanol $(0.07 \pm 0.03 \%)$. This may indicate that, in $M$. acetivorans, either a methyltransferase related to growth on trimethylamine plays a role in methane oxidation, or that methanol is an intermediate of methane oxidation. Addition of possible electron acceptors $\left(\mathrm{O}_{2}, \mathrm{NO}_{3}^{-}, \mathrm{SO}_{4}^{2-}, \mathrm{SO}_{3}^{2-}\right)$ or $\mathrm{H}_{2}$ to the headspace did not substantially enhance or diminish methane oxidation in $M$. acetivorans cultures. Separate growth experiments with FAD and $\mathrm{NAD}^{+}$showed that inclusion of these electron carriers also did not enhance methane oxidation. Our results suggest trace methane oxidized during methanogenesis cannot be coupled to the reduction of these electron acceptors in pure cultures, and that the mechanism by which methane is oxidized in methanogens is independent of $\mathrm{H}_{2}$ concentration. In contrast to the methanogens, species of the sulfate-reducing genus Archaeoglobus did not significantly oxidize methane during growth (oxidizing $0.003 \pm 0.01 \%$ of the methane provided to A. fulgidus, $0.002 \pm 0.009 \%$ to A. lithotrophicus and $0.003 \pm 0.02 \%$ to $A$. profundus). Lack of observable methane oxidation in the three Archaeoglobus species examined may indicate that methyl-coenzyme $\mathrm{M}$ reductase, which is not present in this genus, is required for the anaerobic oxidation of methane, consistent with the "reverse methanogenesis" hypothesis.
\end{abstract}

Keywords: anaerobic methane oxidation, Archaeoglobus, methanogen, reverse methanogenesis, stable isotope label.

\section{Introduction}

The anaerobic oxidation of methane (AOM) is a significant methane sink in marine sediments, oxidizing up to $90 \%$ of the biological methane produced in these environments (Hinrichs et al. 2000). Organisms responsible for AOM (ANME-1 and ANME-2) have not been isolated, but genetic and lipid analyses indicate the methane-consuming organisms are archaeal and closely related to cultured methanogenic genera, specifically Methanosarcina and Methanococcoides (Hinrichs et al. 1999, 2000, Boetius et al. 2000, Orphan et al. 2001a). Lipid analyses and fluorescence in situ hybridization (FISH) suggest that certain bacteria form tight consortia with the archaeal methanotrophs (ANME-2), coupling methane oxidation to sulfate-reduction and rendering the overall reaction thermodynamically favorable (Boetius et al. 2000, Orphan et al. 2001b). Nauhaus et al. (2002), investigating methane seep sediments, showed a 1:1 reduction of sulfate to sulfide with the oxidation of methane to $\mathrm{CO}_{2}$ when methane served as the only available electron donor. The bacterial component of these natural consortia is most closely related to isolated sulfate-reducers from the Desulfosarcinales (Orphan et al. 2001a), and stable isotope evidence suggests their biomass is, at least in part, composed of carbon from oxidized methane (Hinrichs et al. 2000, Pancost et al. 2000, Orphan et al. 2001b, Michaelis et al. 2002). Some archaeal methane-oxidizing organisms (ANME-1) may perform AOM and associated sulfate reduction unaided by bacteria (Michaelis et al. 2002, Orphan et al. 2002).

The enzymatic mechanisms mediating AOM in marine sediments remain unknown. One possible mechanism, proposed by Hoehler et al. (1994), is reverse methanogenesis. In this paper, we refer to "reverse methanogenesis" as the reversal of a methanogen's typical metabolism such that methane production is replaced by net methane oxidation. Hallam et al. (2004) recently found nearly all genes associated with methanogensis in environmental ANME-1 sequences. It is hypothesized that reverse methanogenesis begins with methane activation by either methyl-coenzyme M reductase (MCR) or methyl transfer- 
ase (MT) as described by Hoehler and Alperin (1996). Methyl-coenzyme $M$ reductase catalyzes the final reductive step in all known biogenic methane formation and the operon ( $m c r A$ ) encoding this enzyme is observed in all known methanogen species. Consistent with the reverse methanogenesis hypothesis is the recent discovery of $m c r A$ or a close variant in both ANME-1 and ANME-2 environmental samples (Hallam et al. 2003, 2004, Krüger et al. 2003b). In the event that ANME-2 methanotrophy is initiated by reverse MCR activity, analysis of closely related, culturable species with active MCR may provide a suitable model system for studying archaeal methane oxidation until the indigenous archaeal methanotrophs can be cultured.

We use the term "trace methane oxidation" (TMO) to refer to the conversion of labeled methane to $\mathrm{CO}_{2}$ in methanogen cultures during typical methanogenic growth (Zehnder and Brock 1979, Harder 1997). The extent to which reverse methanogenesis and TMO are related is undetermined. Yet, if the two processes are mechanistically similar, providing an organism capable of TMO with favorable environmental conditions could potentially initiate reverse methanogenesis.

Although never experimentally demonstrated, Hohler and Alperin (1996) used a thermodynamic approach to argue that under the proper conditions, principally low $\mathrm{H}_{2}$ pressures, reverse methanogenesis is favored. Also, exposure to typical methanogenesis inhibitors (such as bromoethane or methyl fluoride) halts AOM activity within marine consortia (Krüger et al. 2003a), suggesting a possible link to methanogenesis pathways. The reverse methanogenesis hypothesis invokes consortia associations to help maintain thermodynamic favorability by consuming the methanotroph's metabolic end product, keeping its concentration below a critical thermodynamic threshold. One possible end product is $\mathrm{H}_{2}$, which could be produced by an archaeal methanotroph and consumed by sulfate-reducing bacteria. Valentine et al. (2000) monitored $\mathrm{H}_{2}$ production rates in stressed methanogen cultures subjected to high methane concentrations and low methanogenic substrate levels. No link between methane abundance and $\mathrm{H}_{2}$ production was observed, suggesting reverse methanogenesis is either unrelated to $\mathrm{H}_{2}$ production or it is not a metabolism adoptable by the methanogens tested. Acetate has been proposed as an alternative AOM intermediate (Valentine and Reeburgh 2000). Yet, Nauhuas et al. (2002) tested multiple potential intermediates (including acetate, formate, hydrogen and methanol) and demonstrated these possible intermediates did not greatly enhance sulfate reduction rates in methane seep sediment samples, suggesting that acetate is not an intermediate for in situ AOM.

In order to further understanding of TMO and evaluate the reverse methanogenesis hypothesis, we examined TMO in pure cultures of three Archaeoglobus species and three methanogen species. Our experiments were aimed at determining (1) if archaeal species more closely related to AOM methanotrophs exhibited a stronger propensity for methane oxidation expressed through enhanced TMO, (2) whether the inclusion of different potential electron acceptors make conditions more amenable to methane oxidation, increasing the level of TMO and (3) if archaeal sulfate-reducers, which share genetic similarity to the ANME group but lack the potential enzymatic methane activators (namely MCR) required for reverse methanogensis, exhibit TMO.

For each experiment, a set of cultures was grown under headspaces containing differing proportions of ${ }^{13} \mathrm{C}$-labeled methane. Following growth, isotopic analysis was used to quantify the amount of ${ }^{13} \mathrm{C}$ label converted from methane to $\mathrm{CO}_{2}$, indicating the extent of TMO in the culture.

\section{Materials and methods}

\section{Microorganisms}

Archaeoglobus fulgidus VC16, Archaeoglobus profundus $\mathrm{AV} 18$ and Methanobacterium thermoautotrophicum $\Delta \mathrm{H}$ were obtained from the Deutsche Sammlung von Mikroorganismen und Zellkulturen (DSMZ, Braunschweig, Germany). Methanosarcina barkeri MS was obtained from the Oregon Collection of Methanogens (Portland, OR). Archaeoglobus lithotrophicus TF2 was a kind gift from Karl Stetter (emeritus, Universität Regensburg, Germany). Methanosarcina acetivorans 2CA was provided by J.G.F.

\section{Media}

Strict anaerobic technique was employed for cell culturing. Media for A. fulgidus and A. profundus were prepared as recommended by DSMZ (media 399 and 519, respectively). Methanobacterium thermoautotrophicum was cultured on medium containing: $3.0 \mathrm{~g} \mathrm{Na}_{2} \mathrm{SO}_{4}, 0.2 \mathrm{~g} \mathrm{KH}_{2} \mathrm{PO}_{4}, 0.3 \mathrm{~g} \mathrm{NH}{ }_{4} \mathrm{Cl}$, $0.3 \mathrm{~g} \mathrm{KCl}, 0.2 \mathrm{~g} \mathrm{CaCl}_{2} \cdot 2 \mathrm{H}_{2} \mathrm{O}, 0.3 \mathrm{~g} \mathrm{MgCl}_{2} \cdot 6 \mathrm{H}_{2} \mathrm{O}, 1.0 \mathrm{ml} 10 \times$ Wolfe solution (Wolin et al. 1963), $0.5 \mathrm{mg}$ resazurin, $3.0 \mathrm{ml}$ $\mathrm{NaOH}$ solution (10\%) and $0.5 \mathrm{~g} \mathrm{Na}_{2} \mathrm{~S} \cdot 9 \mathrm{H}_{2} \mathrm{O}$. All reagents except $\mathrm{Na}_{2} \mathrm{~S}$ were mixed in solution and degassed by bubbling with $\mathrm{N}_{2}$ for 20 min followed by $\mathrm{Na}_{2} \mathrm{~S}$ addition. The medium ( $20 \mathrm{ml}$ per bottle) was dispensed into 120 -ml culture bottles in an anaerobic chamber followed by three flushes with a mixture of $\mathrm{H}_{2}$ and $\mathrm{CO}_{2}(80: 20(\mathrm{v} / \mathrm{v}))$ and subsequent filling of the bottles to $0.3 \mathrm{MPa}$ pressure with the gas mixture and sterilization in an autoclave. Archaeoglobus lithotrophicus was cultured in the same medium, but with the addition of $20 \mathrm{~g} \mathrm{NaCl}^{-1}$. Methanosarcina barkeri was cultured in medium containing (per liter): $0.29 \mathrm{~g} \mathrm{~K}_{2} \mathrm{HPO}_{4} \cdot 3 \mathrm{H}_{2} \mathrm{O}, 0.23 \mathrm{~g} \mathrm{KH}_{2} \mathrm{PO}_{4}, 0.23 \mathrm{~g}$ $\left(\mathrm{NH}_{4}\right)_{2} \mathrm{SO}_{4}, \quad 0.45 \mathrm{~g} \mathrm{NaCl}, 0.06 \mathrm{~g} \mathrm{CaCl}_{2} \cdot 2 \mathrm{H}_{2} \mathrm{O}, 0.09 \mathrm{~g}$ $\mathrm{MgSO}_{4} \cdot 7 \mathrm{H}_{2} \mathrm{O}, 1.0 \mathrm{ml}\left(\mathrm{NH}_{4}\right)_{2} \mathrm{Ni}\left(\mathrm{SO}_{4}\right)_{2}$ solution $(0.2 \%), 1.0 \mathrm{ml}$ $\mathrm{FeSO}_{4} \cdot 7 \mathrm{H}_{2} \mathrm{O}$ solution $(0.2 \%), 0.5 \mathrm{mg}$ resazurin, $1.0 \mathrm{ml} 10 \times$ Wolfe mineral solution (Wolin et al. 1963), $6.0 \mathrm{ml}$ vitamin solution, $4.0 \mathrm{ml} \mathrm{NaOH}$ solution (10\%) and prepared as above with the addition of $0.5 \mathrm{~g} \mathrm{l}^{-1} \mathrm{Na}_{2} \mathrm{~S} \cdot 9 \mathrm{H}_{2} \mathrm{O}$. Medium for $M$. acetivorans contained (per liter final solution): Mix A: $23.4 \mathrm{~g} \mathrm{NaCl}, 3.8 \mathrm{~g} \mathrm{NaHCO}_{3}, 1.0 \mathrm{~g} \mathrm{KCl}, 1.0 \mathrm{ml} 10 \times$ Wolfe mineral solution, $2.0 \mathrm{ml}$ vitamin solution and $0.5 \mathrm{mg}$ resazurin; Mix B: $11.0 \mathrm{~g} \mathrm{MgCl}_{2} \cdot 6 \mathrm{H}_{2} \mathrm{O}, 0.30 \mathrm{~g} \mathrm{CaCl}_{2} \cdot 2 \mathrm{H}_{2} \mathrm{O}$ and $0.68 \mathrm{~g}$ $\mathrm{KH}_{2} \mathrm{PO}_{4}$; Mix C: $1.0 \mathrm{~g} \mathrm{NH}_{4} \mathrm{Cl}, 0.50 \mathrm{~g}$ cysteine hydrochloride and appropriate substrate; and Mix D: $0.50 \mathrm{~g} \mathrm{Na} 2 \mathrm{~S} \cdot 9 \mathrm{H}_{2} \mathrm{O}$. Mixes A and B in $500 \mathrm{ml}$ water each were degassed under $\mathrm{N}_{2}$ then combined in an anaerobic chamber followed by addition of Mix C and finally the addition of Mix D. Substrates were 
trimethylamine $\left(9.56 \mathrm{~g} \mathrm{l}^{-1}\right)$ or methanol $\left(5.0 \mathrm{ml} \mathrm{l}^{-1}\right)$. Once dispensed ( $30 \mathrm{ml}$ per culture bottle), individual bottles were flushed three times, then filled to 0.2 MPa pressure with a mixture of $\mathrm{N}_{2}$ and $\mathrm{CO}_{2}(80: 20(\mathrm{v} / \mathrm{v}))$ and autoclaved. For the experiment involving $M$. acetivorans and $\mathrm{H}_{2}$, the culture bottles were pressurized to $0.2 \mathrm{MPa}$ with a mixture of $\mathrm{H}_{2}$ and $\mathrm{CO}_{2}$ $(80: 20(\mathrm{v} / \mathrm{v}))$. For experiments involving M. acetivorans and oxygen, neither $\mathrm{Na}_{2} \mathrm{~S}$ nor resazurin were included in the growth media and sterile oxygen was injected into the bottles after autoclaving.

\section{Cultivation}

Two sets of four culture bottles were used for each species investigated. A total of $40 \mathrm{ml}$ (at $0.1 \mathrm{MPa} \approx 1.8 \mathrm{mmol}$ ) of methane was added to each culture bottle with increasing proportions of labeled methane $\left(99 \%{ }^{13} \mathrm{CH}_{4}, 1 \%{ }^{12} \mathrm{CH}_{4}\right)$ added to each bottle in the series (Lot 00-04, Cambridge Isotope Labs, Cambridge, MA). For each tested species, one set of bottles was inoculated for growth and the other was kept uninoculated as a negative control. All species, except $M$. barkeri, were incubated for 1 week to ensure maximum growth and substrate consumption. Because of its slower growth, M. barkeri was incubated for 2 months. The amount of methane produced in each culture was calculated from established stoichiometric relationships for each substrate and final methane production. The cultures were grown to completion, ensuring complete substrate usage. Measurement of methane concentration in parallel experiments, by gas chromatography with a thermal conductivity detector, confirmed the accuracy of calculated methane productivity. Cell counts following growth were performed by phase- contrast microscopy with a counting chamber for all species but $M$. barkeri where cell clumping prevented accurate counting.

\section{Sample preparation}

Following culture growth, two distillations were performed to collect and purify $\mathrm{CO}_{2}$ from each sample for isotope analysis. The first distillation was done with an apparatus (Figure 1) we developed for collection of $\mathrm{CO}_{2}$ from high pressure cultures with up to $0.4 \mathrm{MPa}$ of mixed gases. After growth, a sample bottle was connected to the evacuated distilling apparatus through a gas-tight needle. A liquid nitrogen trap froze $\mathrm{CO}_{2}$ from the culture bottles into a separate collection tube, and gaseous methane was pumped from the sample. A secondary liquid nitrogen trap ensured quantitative $\mathrm{CO}_{2}$ collection. During growth, the Archaeoglobus spp. produced $\mathrm{H}_{2} \mathrm{~S}$, which interfered with preliminary $\mathrm{CO}_{2}$ isotope analysis. For these samples, about $100 \mathrm{mg}$ of silver foil was included in the sample collection vial at room temperature over $24-48 \mathrm{~h}$ prior to analysis on the mass spectrometer. The foil removed all $\mathrm{H}_{2} \mathrm{~S}$ artifacts from the isotopic analysis. A second distillation cryogenically trapped and removed water (2-propanol and dry ice) and condensed $\mathrm{CO}_{2}$ (liquid nitrogen) to assure residual noncondensable gasses were removed. Isotope analysis to determine the ${ }^{13} \mathrm{C} /{ }^{12} \mathrm{C}$ ratio for the $\mathrm{CO}_{2}$ in each culture bottle was performed with a Finnigan MAT 252 dual inlet mass spectrometer (Thermo Finnigan, Bremen, Germany).

\section{Data analysis}

Isotope data were plotted as the ${ }^{13} \mathrm{C}$ fractional abundance of the initial methane $\left(F_{\mathrm{CH} 4}={ }^{13} \mathrm{C} /\left({ }^{13} \mathrm{C}+{ }^{12} \mathrm{C}\right)\right)$ in each culture bottle versus the measured fractional abundance of $\mathrm{CO}_{2}\left(F_{\mathrm{CO} 2}\right)$ in each bottle after incubation (Figure 2). Least-squares calculations provide the slope and intercept for the resulting relationships and a positive slope indicates methane oxidation. We next consider mass balance for methane and $\mathrm{CO}_{2}$ produced by $M$. acetivorans cultures during growth on either trimethylamine or methanol. The methane in a culture bottle after growth represents that contributed during growth and methane lost by oxidation:

$$
F_{\mathrm{T}} n_{\mathrm{T}}=F_{\mathrm{i}} n_{\mathrm{i}}+F_{\mathrm{b}} n_{\mathrm{b}}-F_{\mathrm{ox}} n_{\mathrm{ox}}
$$

where $F_{\mathrm{T}}, F_{\mathrm{i}}, F_{\mathrm{b}}$ and $F_{\text {ox }}$ represent the fractional abundance of carbon isotopes in total, initial, biogenic and oxidized methane, respectively. Likewise, $n_{\mathrm{T}}, n_{\mathrm{i}}, n_{\mathrm{b}}$ and $n_{\mathrm{ox}}$ represent the moles of total, initial, biogenic and oxidized methane, respectively. We assume that any fractionation in the methane oxidation process is insignificant in our strongly labeled system, such that $F_{\mathrm{ox}}=F_{\mathrm{T}}$; we can therefore simplify Equation 1 and solve for $F_{\mathrm{T}}$ :

$$
F_{\mathrm{T}}=\frac{F_{\mathrm{i}} n_{\mathrm{i}}+F_{\mathrm{b}} n_{\mathrm{b}}}{n_{\mathrm{i}}+n_{\mathrm{b}}}
$$

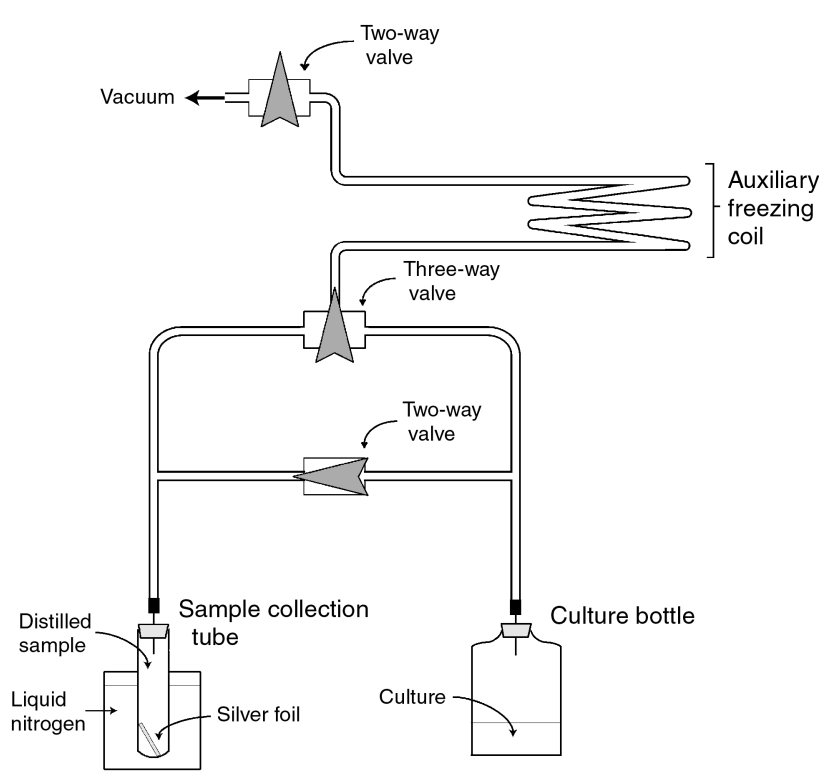

Figure 1 . The distilling apparatus used to isolate $\mathrm{CO}_{2}$ cryogenically from pressurized culture bottles after growth. Sample was introduced to the system through a needle. Liquid nitrogen collected $\mathrm{CO}_{2}$ in a separate sample tube, allowing for subsequent removal of non-condensable gases by a vacuum. The auxiliary freezing coil was also cooled by liquid nitrogen and served as a secondary trap for assuring no $\mathrm{CO}_{2}$ loss. Heating the auxiliary freezing coil after removing noncondensable gases allowed any trapped $\mathrm{CO}_{2}$ to migrate to the sample tube. 
We assume oxidized $\mathrm{CH}_{4}$ is converted to $\mathrm{CO}_{2}$, such that:

$$
F_{\mathrm{T}_{\mathrm{CO} 2}} n_{\mathrm{T}_{\mathrm{CO} 2}}=F_{\mathrm{i}_{\mathrm{CO} 2}} n_{\mathrm{i}_{\mathrm{CO} 2}}+F_{\mathrm{b}_{\mathrm{CO} 2}} n_{\mathrm{b}_{\mathrm{CO} 2}}+F_{\mathrm{T}_{\mathrm{CH} 4}} n_{\mathrm{ox}}
$$

where $F_{\mathrm{T}_{\mathrm{CO} 2}}, F_{\mathrm{i}_{\mathrm{CO} 2}}$ and $F_{\mathrm{b}}$ ro2 represent fractional abundance of carbon isotopes in total, initial and biogenic $\mathrm{CO}_{2}$, respectively. Similarly, $n_{\mathrm{T}_{\mathrm{CO} 2}}, n_{\mathrm{i}_{\mathrm{CO} 2}}$ and $n_{\mathrm{b}_{\mathrm{CO} 2}}$ represent moles of total, initial and biogenic $\mathrm{CO}_{2}$, respectively. In a culture bottle containing unlabeled methane, we can ignore the methane oxidation input into the $\mathrm{CO}_{2}$ mass balance because the small $n_{\mathrm{ox}}$ does not significantly affect $F_{\mathrm{T}_{\mathrm{CO} 2}}$ or $n_{\mathrm{T}_{\mathrm{CO} 2}}$. In cases with unlabeled methane we have:

$$
F_{\mathrm{T}_{\mathrm{CO} 2}^{*}} n_{\mathrm{T}_{\mathrm{CO} 2} *}=F_{\mathrm{C}^{*}{ }^{*}} n_{\mathrm{i}_{\mathrm{CO}^{*}}}+F_{\mathrm{b}^{*} 2^{*}} n_{\mathrm{b}_{\mathrm{CO} 2} *}
$$

where terms are defined as in Equation 3, and an asterisk refers to bottles cultured with unlabeled methane. We prepared incubation bottles such that $F_{\mathrm{i}_{\mathrm{CO} 2}}=F_{\mathrm{i}_{\mathrm{CO} 2}{ }^{*}}$ and $n_{\mathrm{i}_{\mathrm{CO} 2}}=n_{\mathrm{i}_{\mathrm{CO} 2}{ }^{*}}$. Therefore, unlabeled incubations account for $\mathrm{CO}_{2}$ produced during cell growth:

$$
F_{\mathrm{T}_{\mathrm{CO} 2}} n_{\mathrm{T}_{\mathrm{CO} 2}}=F_{\mathrm{T}_{\mathrm{CO} 2} *} n_{\mathrm{T}_{\mathrm{CO} 2} *}+F_{\mathrm{T}} n_{\mathrm{ox}}
$$

Combining Equations 2 and 5 and solving for $n_{\text {ox }}$ gives:

$$
n_{\mathrm{ox}}=\left(F_{\mathrm{T}_{\mathrm{CO} 2}} n_{\mathrm{T}_{\mathrm{CO} 2}}-F_{\mathrm{T}_{\mathrm{CO} 2} *} n_{\mathrm{T}_{\mathrm{CO} 2} *}\right) \frac{F_{\mathrm{i}} n_{\mathrm{i}}+F_{\mathrm{b}} n_{\mathrm{b}}}{n_{\mathrm{i}}+n_{\mathrm{b}}}
$$

where $F_{\mathrm{T}_{\mathrm{CO} 2}}$ and $F_{\mathrm{T}_{\mathrm{CO}_{2}} *}$ were measured in multiple sample cultures with varied $F_{\mathrm{i}}$ (Figure 2 ). The value of $F_{\mathrm{T}_{\mathrm{C} 2}}$ was interpolated from the linear regression of the data at the median $F_{\mathrm{i}}$, whereas $F_{\mathrm{T}_{\mathrm{Co} 2} *}$ was interpolated from the same line at the point with no labeled methane. We controlled the amount of labeled methane added to each bottle, with $n_{\mathrm{i}}$ fixed at $1.8 \mathrm{mmol}$. We estimated the biogenic methane composition to be $\delta=-50 \%$, and therefore $F_{\mathrm{b}}=0.0106$. Because of the strong label, small variations in $F_{\mathrm{b}}$ become inconsequential to the results. The value of $n_{\mathrm{b}}$ was estimated based on the stoichiometry of methanogenesis for a given substrate and confirmed in parallel experiments by gas chromatography with a thermal conductivity

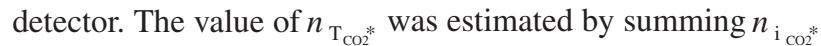
and $n_{\mathrm{b}_{\mathrm{Co}_{2}^{*}}} ; \mathrm{CO}_{2}$ input from $\mathrm{CH}_{4}$ oxidation was small in comparison with other $\mathrm{CO}_{2}$ sources.

We solved for $n_{\mathrm{ox}}$ using an iterative method. First, $n_{\mathrm{T}_{\mathrm{CO} 2}}$ was estimated as equal to $n_{\mathrm{T}_{\mathrm{CO}}{ }^{*}}$. We then solved for $n_{\mathrm{ox}}$ by adjusting $n_{\mathrm{T}_{\mathrm{CO} 2}}$ to account for $\mathrm{CO}_{2}$ derived from $\mathrm{CH}_{4}$ oxidation. The $n_{\mathrm{ox}}$ values converged within two to three iterations. We calculated $n_{\mathrm{ox}}$ for both the growth experiments and control bottles (media with labeled $\mathrm{CH}_{4}$ but no cells) and the amount of $\mathrm{CH}_{4}$ oxidized in each experiment is reported as the difference between these two values. Error estimates are based on the standard error of the slope used to calculated $F_{\mathrm{T}_{\mathrm{CO} 2}}$ and $F_{\mathrm{T}_{\mathrm{CO} 2} *}$. Final reported error was estimated by repeating the above calculations using an experimental slope plus twice the slope standard error (i.e., 2 SD) and a control slope minus twice the slope standard error. The same system of calculations was used for determining $\mathrm{CH}_{4}$ oxidation in the other methanogen and sul-
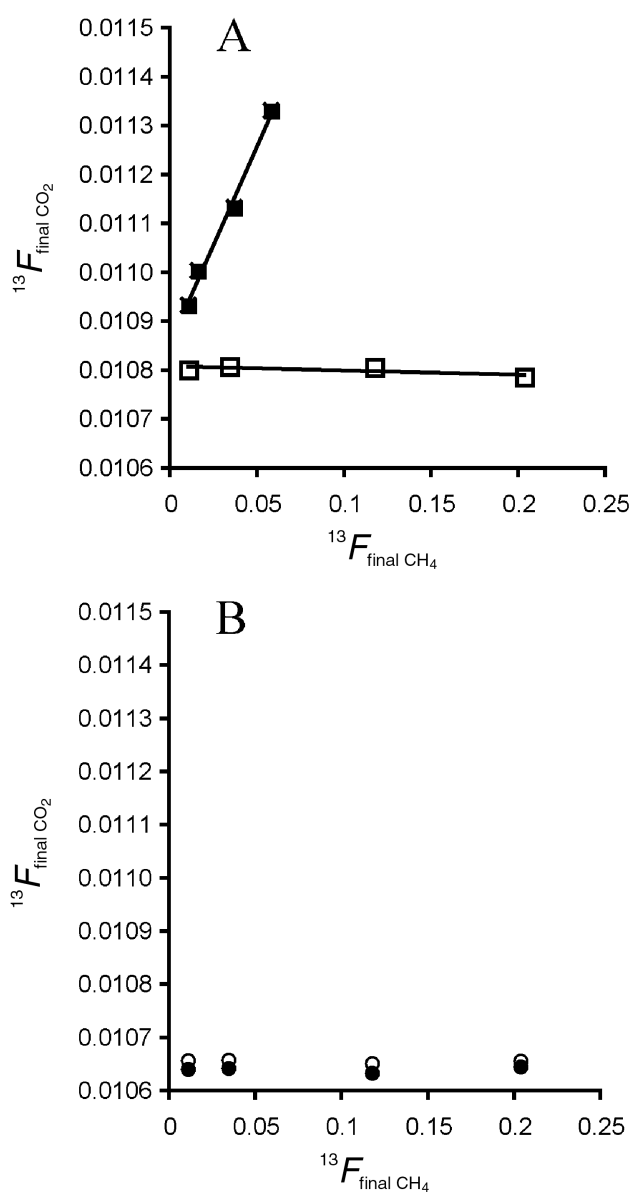

Figure 2. The ${ }^{13} \mathrm{C}$ fractional abundance $\left({ }^{13} \mathrm{~F}\right)$ of a culture bottle's $\mathrm{CO}_{2}$ after incubation versus ${ }^{13} \mathrm{~F}$ of final $\mathrm{CH}_{4}$ tracks methane oxidation to $\mathrm{CO}_{2}$. (A) The ${ }^{13} \mathrm{FCO}_{2}$ in culture bottle headspace following incubation versus ${ }^{13} \mathrm{~F}$ of the final methane in each bottle for Methanosarcina acetivorans cultures $(\boldsymbol{\square})$ grown with trimethylamine and for identical, but not inoculated, control bottles ( $\square$ ). The difference in slopes between the cultured $\left(8.0 \times 10^{-3}\right)$ and control samples $\left(-8.6 \times 10^{-5}\right)$ determined the amount of methane oxidation in the experiment. (B) Results from Archaeoglobus profundus $(\bullet)$ and control samples $(\bigcirc)$ showing no direct relationship between the extent of final methane enrichment and ${ }^{13} \mathrm{~F}$ of final $\mathrm{CO}_{2}$, indicating no methane oxidation by the cultures.

fate reducer cultures, adjusted to account for stoichiometric input (or uptake) of biogenic methane and $\mathrm{CO}_{2}\left(n_{\mathrm{b}}, n_{\mathrm{b}_{\mathrm{CO} 2}}\right)$. Methane oxidation is reported as both the percent of total postgrowth methane oxidized in each bottle and, for methanogens, as the percent of methane produced during growth that was oxidized to $\mathrm{CO}_{2}$.

\section{Results and discussion}

Archaeoglobus is an archaeal sulfate-reducing genus with multiple culturable representatives (Stetter et al. 1987, 1993). There are several reasons we chose to look at Archaeoglobus for studying TMO. First, recent environmental genomic sequencing of uncultured AOM methanotrophs revealed a gen- 
eral core similarity between the ANME-1 and the Archaeoglobales (Hallam et al. 2004). Second, sulfate reduction by Archaeoglobus could provide an electron sink for methane oxidation. Third, Archaeoglobus species possess much of the enzymatic machinery associated with methanogenesis (Klenk et al. 1998). Archaeoglobus fulgidus, for example, contains enzymes involved with the reduction of $\mathrm{CO}_{2}$ to a methyl group in methanogenesis, but operates these enzymes in the reverse direction, oxidizing methyl groups to $\mathrm{CO}_{2}$. Yet, Archaeoglobus species lack MCR (Stetter et al. 1987, Klenk et al. 1998), used in all known methanogenic pathways and thought likely to be required for reverse methanogenesis. Although the role of MCR in methanotrophy is undetermined, its absence from Archaeoglobus species would prohibit the initiation steps required for reverse methanogenesis unless an alternative enzymatic pathway for methane activation is present. We examined methane oxidation in three Archaeoglobus species, each utilizing different growth substrates for sulfate reduction: A. fulgidus utilizes lactate; $A$. profundus utilizes acetate and $\mathrm{H}_{2}$; and A. thermolithotrophicus grows chemolithotrophically on media containing $\mathrm{H}_{2}$ and $\mathrm{CO}_{2}$. In each case, the amount of methane oxidized was not detected within the experimental error (Figure 3, Table 1). Lack of observable TMO in these species suggests MCR is required for TMO, which is consistent with the reverse methanogenesis hypothesis for AOM.

The methanogens, Mb. thermoautotrophicum, M. barkeri and $M$. acetivorans, each displayed small amounts of methane oxidation (Figure 3, Table 1), consistent with previous reports based on similar experimental work (Zehnder and Brock 1979, 1980, Harder 1997). We further explored the reaction in $M$. acetivorans, which has a close rRNA phylogenetic relationship with the ANME-2 methanotroph lineage (Orphan et al. 2001a) and also has a large and complex genome ( 5.8 Mb) with high metabolic diversity (Galagan et al. 2002). Such a close relationship to indigenous AOM species coupled with its large genome makes $M$. acetivorans a reasonable candidate for harboring genes required for reverse methanogenesis and may make it a valuable model organism for studying archaeal methane oxidation. The TMO we observed suggests a path- way for methane oxidation is present in $M$. acetivorans.

We tested whether $\mathrm{O}_{2}, \mathrm{NO}_{3}^{-}, \mathrm{SO}_{4}^{2-}$ and $\mathrm{SO}_{3}^{2-}$ could be used as electron acceptors in methane oxidation by $M$. acetivorans. Enriching the culture medium with these electron acceptors could stimulate methane oxidation if electrons can be transferred to them effectively. Sulfite is a metabolic intermediate in bacterial sulfate reduction and, therefore, we hypothesize that it could be transferred between the archaeal and bacterial members of in vivo AOM consortia. In our model, sulfite transfer within the AOM consortia from the sulfate reducer to the archaeal ANME-2 would provide oxidizing potential for the methanotrophic reactions. However, none of the electron acceptors tested with $M$. acetivorans substantially enhanced the amount of methane oxidized in the cultures (Figure 3, Table 1), indicating that these electron acceptors, including sulfite, are not utilized by this methanogen to oxidize methane, even in trace amounts.

Interestingly, most methanogen species are extremely oxygen sensitive, being killed by even trace amounts of oxygen. However, two superoxide dismutases, a superoxide reductase and a catalase, were uncovered in the $M$. acetivorans genome, suggesting the potential for oxygen resistance (Galagan et al. 2002). The presence of a cytochrome $d$ oxidase may even provide a link between oxygen and energy conservation by the organism (Galagan et al. 2002). We cultured M. acetivorans under a microaerophilic headspace $\left(0.7 \% \mathrm{O}_{2}\right)$. Diffusional constraints likely lowered the percent of oxygen in the actual liquid growth medium, but $M$. acetivorans still demonstrated oxygen tolerance under microaerophilic conditions. The presence of oxygen had little effect on cell yields, methane production or trace methane oxidation in these cultures (Figure 3, Table 1), indicating that the oxygen reduction, which is likely occurring, cannot be coupled to methane oxidation. Research is now underway to more closely examine the responses of $M$. acetivorans to oxygen.

We also tested the effect of including $\mathrm{H}_{2}$ in the culture headspace. Methanosarcina acetivorans is capable of growth and methanogenesis using a large array of substrates but does not use $\mathrm{H}_{2}$. Unlike the other methanogens tested, M. aceti-
$\mathrm{CH}_{4}$ oxidation as a percent of total $\mathrm{CH}_{4}$ $\square \mathrm{CH}_{4}$ oxidation as a percent of culture-produced $\mathrm{CH}_{4}$

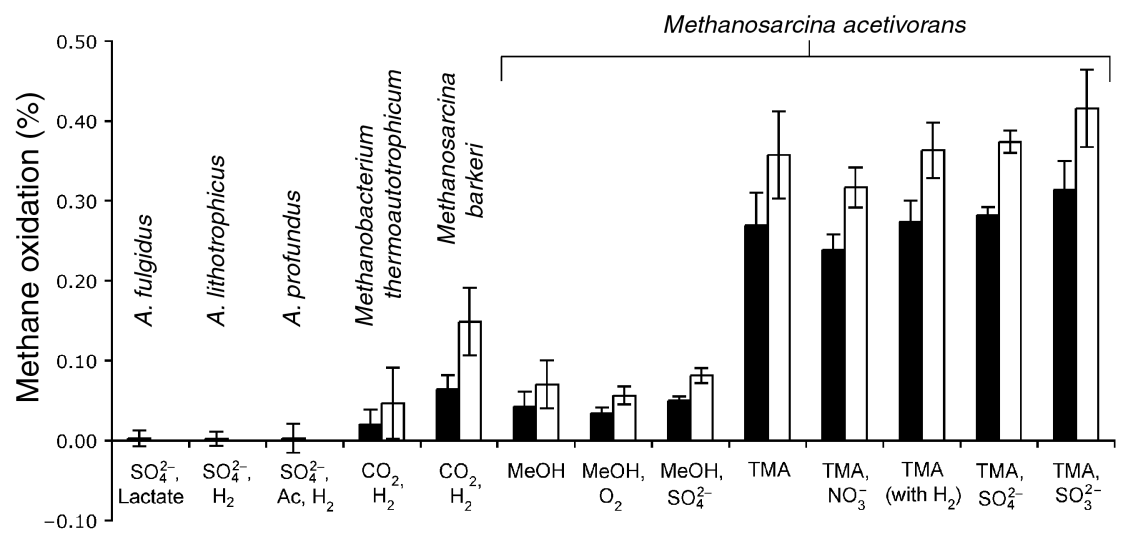

Figure 3. Methane oxidation ( $\pm 2 \mathrm{SD})$ standardized to either the total amount of methane in the culture after growth (black bars) or the methane produced by the culture during growth (white bars) observed in experiments with varying species and substrates. Added concentrations: lactate, $13.4 \mathrm{mM} ; \mathrm{H}_{2}$ (for all but $M$. acetivorans experiment), $0.24 \mathrm{MPa} ; \mathrm{H}_{2}$ (M. acetivorans experiment), $0.16 \mathrm{MPa}$; Ac (acetate), $12.2 \mathrm{mM} ; \mathrm{SO}_{4}^{2-}$ (for A. fulgidus), $14.0 \mathrm{mM} ; \mathrm{SO}_{4}^{2-}$ (for A. profundus), $33.0 \mathrm{mM} ; \mathrm{SO}_{4}^{2-}$ (for A. thermolithotrophicus), $21.1 \mathrm{mM} ; \mathrm{MeOH}$ (methanol), $125 \mathrm{mM} ; \mathrm{O}_{2}, 0.7 \mathrm{kPa}$; TMA (trimethylamine), $100 \mathrm{mM} ; \mathrm{NO}_{3}^{-}, 6.59 \mathrm{mM}$; $\mathrm{SO}_{4}^{2-}$ (for M. acetivorans experiments), $21.1 \mathrm{mM}$; and $\mathrm{SO}_{3}^{2-}, 2 \mathrm{mM}$. 
Table 1. Growth conditions and methane oxidation per culture experiment. Each culture was initially provided $1.79 \mathrm{E}-3$ mol of methane. In addition, based on methanogenesis, stoichiometry and measurements performed in parallel experiments, the cultures produced $1.3 \mathrm{E}-3,2.8 \mathrm{E}-3$ and $5.5 \mathrm{E}-3 \mathrm{~mol}$ of methane for the cultures grown on $\mathrm{H}_{2} / \mathrm{CO}_{2}$, methanol and trimethylamine (TMA), respectively.

\begin{tabular}{|c|c|c|c|c|c|c|c|}
\hline Species & $\begin{array}{l}\text { Growth } \\
\text { substrate }\end{array}$ & $\begin{array}{l}\text { Media } \\
\text { addition }\end{array}$ & $\begin{array}{l}\text { Incubation } \\
\text { temperature }\left({ }^{\circ} \mathrm{C}\right)\end{array}$ & $\begin{array}{l}\text { Cell count } \\
\left(\mathrm{ml}^{-1}\right)\end{array}$ & $\begin{array}{l}\text { Experimental } \\
\text { slope }\end{array}$ & $\begin{array}{l}\text { Control } \\
\text { slope }\end{array}$ & $\begin{array}{l}\text { Methane oxidized } \\
(\mathrm{mol})\end{array}$ \\
\hline A. fulgidus & Lactate & - & 85 & $2 \mathrm{E}+8$ & $3.1 \mathrm{E}-5$ & $1.7 \mathrm{E}-5$ & $4.7 \mathrm{E}-8( \pm 1.7 \mathrm{E}-7)$ \\
\hline A. lithotrophicus & $\mathrm{H}_{2}, \mathrm{CO}_{2}$ & - & 85 & $7 \mathrm{E}+7$ & $9.7 \mathrm{E}-5$ & $8.1 \mathrm{E}-5$ & $3.8 \mathrm{E}-7( \pm 1.6 \mathrm{E}-7)$ \\
\hline A. profundus & Acetate, $\mathrm{H}_{2}$ & - & 85 & $1 \mathrm{E}+8$ & $7.8 \mathrm{E}-6$ & $-1.3 \mathrm{E}-5$ & $5.2 \mathrm{E}-8( \pm 2.3 \mathrm{E}-7)$ \\
\hline Mb. thermoautotrophicum & $\mathrm{H}_{2}, \mathrm{CO}_{2}$ & - & 65 & $5 \mathrm{E}+7$ & $6.1 \mathrm{E}-4$ & $2.8 \mathrm{E}-5$ & $6.3 \mathrm{E}-7( \pm 5.9 \mathrm{E}-7)$ \\
\hline M. barkeri & $\mathrm{H}_{2}, \mathrm{CO}_{2}$ & - & 33.5 & $\mathrm{n} / \mathrm{a}^{1}$ & $1.8 \mathrm{E}-3$ & $6.0 \mathrm{E}-6$ & $2.0 \mathrm{E}-6( \pm 5.7 \mathrm{E}-7)$ \\
\hline M. acetivorans & Methanol & - & 33.5 & $5 \mathrm{E}+8$ & $1.0 \mathrm{E}-3$ & $4.7 \mathrm{E}-5$ & $2.0 \mathrm{E}-6( \pm 8.5 \mathrm{E}-7)$ \\
\hline M. acetivorans & Methanol & $\mathrm{O}_{2}$ & 33.5 & $5 \mathrm{E}+8$ & $7.9 \mathrm{E}-4$ & $1.2 \mathrm{E}-5$ & $1.6 \mathrm{E}-6( \pm 3.2 \mathrm{E}-7)$ \\
\hline M. acetivorans & Methanol & $\mathrm{SO}_{4}^{2-}$ & 33.5 & $4 \mathrm{E}+8$ & $1.1 \mathrm{E}-3$ & $4.9 \mathrm{E}-6$ & $2.3 \mathrm{E}-6( \pm 2.6 \mathrm{E}-7)$ \\
\hline M. acetivorans & TMA & - & 33.5 & $1 \mathrm{E}+9$ & $8.0 \mathrm{E}-3$ & $-8.6 \mathrm{E}-5$ & $2.0 \mathrm{E}-5( \pm 3.0 \mathrm{E}-6)$ \\
\hline M. acetivorans & TMA & $\mathrm{NO}_{3}^{-}$ & 33.5 & $9 \mathrm{E}+8$ & $7.2 \mathrm{E}-3$ & $9.2 \mathrm{E}-5$ & $1.7 \mathrm{E}-5( \pm 1.4 \mathrm{E}-6)$ \\
\hline M. acetivorans & TMA & $\mathrm{H}_{2}$ & 33.5 & $2 \mathrm{E}+9$ & $8.3 \mathrm{E}-3$ & $7.0 \mathrm{E}-5$ & $2.0 \mathrm{E}-5( \pm 1.9 \mathrm{E}-6)$ \\
\hline M. acetivorans & TMA & $\mathrm{SO}_{4}^{2-}$ & 33.5 & $5 \mathrm{E}+8$ & $8.5 \mathrm{E}-3$ & $3.4 \mathrm{E}-5$ & $2.0 \mathrm{E}-5( \pm 7.6 \mathrm{E}-7)$ \\
\hline M. acetivorans & TMA & $\mathrm{SO}_{3}^{2-}$ & 33.5 & $1 \mathrm{E}+9$ & $9.4 \mathrm{E}-3$ & $-2.5 \mathrm{E}-5$ & $2.3 \mathrm{E}-5( \pm 2.7 \mathrm{E}-6)$ \\
\hline
\end{tabular}

${ }^{1}$ Clumping of $M$. barkeri during growth prevented accurate cell counting for these cultures.

vorans lacks two essential hydrogenases required for methanogenic growth using hydrogen but does have other hydrogenases frequently associated with methanogenesis (Galagan et al. 2002). Hydrogen transfer between AOM consortia members may be essential for maintaining the low hydrogen concentrations required by thermodynamic constraints (Hoehler et al. 1994). In this experiment, however, the presence of hydrogen did not have a deleterious effect on the amount of methane oxidized (Figure 3, Table 1).

Nauhaus et al. (2002) suggested that AOM depends on the transfer of electron carriers between consortia members. In this model, ANME-2 would donate electrons from methane to an oxidized electron carrier that is then passed to the bacterial sulfate-reducer. We used a simplified protocol to determine whether adding either $\mathrm{NAD}^{+}$or FAD, the oxidized forms of these carriers, to $M$. acetivorans cultures increased methane oxidation during growth. We incubated cultures with trimethylamine (TMA) as a growth substrate and also provided labeled methane (initial $F_{\mathrm{CH} 4}$ estimated at 0.117 ) in media containing $0.1 \mathrm{mM} \mathrm{NAD}^{+}$or FAD. If these common electron carriers can be used by $M$. acetivorans during TMO we would have observed enhanced methane oxidation. Yet, when compared to the results shown in Table 1 , neither $\mathrm{NAD}^{+}$nor FAD elevated methane oxidation in $M$. acetivorans above typical background values, suggesting the two electron carriers cannot be coupled to methane oxidation.

We also examined $M$. acetivorans for trace methane oxidation when grown on two different substrates, methanol and TMA. More methane was oxidized when TMA, versus methanol, was the substrate for methanogensis. The reason for enhanced methane oxidation in cultures grown on TMA is at present unknown. One potential explanation involves enzymatic differences in the TMA and methanol pathways. Methylamine-based methanogenesis requires three distinct methyltransferase I isozymes specific for mono-, di- and trimethylamines, and only one of two isozymes of methyltrans- ferase II. Methanogenesis from methanol uses only one methyltransferase I and one methyltransferase II, both specific for methanol (Ding et al. 2002). Enhanced TMO in cultures grown with TMA may result from enhanced expression of a methyltransferase involved in TMA metabolism or enhanced expression of a closely related homologue of such a methyltransferase. Alternatively, it is possible that methanol may be an end product of TMO. Nauhaus et al. (2002) explored sulfate reduction in marine AOM samples. They found that when methane was removed and replaced by methanol, sulfate reduction continued at approximately half the original rate. Microbial responses to methanol can be sensitive to concentration (for example, methanol becomes toxic at higher concentrations) and adjusting the methanol concentration used by Nauhuas et al. (2002) may optimize the reactions they observed, increasing sulfate reduction. The ability of consortia of sulfate-reducing bacteria to use methanol as a substrate at all suggests that methanol is an intermediate of AOM. The sulfate-reducing bacteria would be crucial for removing excess methanol, preventing its build up from making AOM energetically unfavorable. If a similar mechanism of methane oxidation occurred in our experiments, TMO might be hindered in cultures grown with methanol because high methanol concentrations could limit methane oxidation.

\section{Acknowledgments}

We thank Dr. Michael Arthur for the use of his mass spectrometer and Denny Walizer for invaluable technical assistance. Graduate support for this project was provided by the Penn State Biogeochemical Research Initiative for Education (BRIE) funded by NSF (IGERT) grant DGE-9972759. This work was also funded by the Penn State Astrobiology Research Center (through the National Astrobiology Institute), the National Science Foundation (MCB-0348492) and seed grants from the Center for Environmental Chemistry and Geochemistry and the Ettinger Foundation. 


\section{References}

Boetius, A., K. Ravenschlag, C.J. Schubert et al. 2000. A marine microbial consortium apparently mediating anaerobic oxidation of methane. Nature 407:623-626.

Ding, Y.H., S.P. Zhang, J.F. Tomb and J.G. Ferry. 2002. Genomic and proteomic analyses reveal multiple homologs of genes encoding enzymes of the methanol:coenzyme M methyltransferase system that are differentially expressed in methanol- and acetate-grown Methanosarcina thermophila. FEMS Microbiol. Lett. 215: 127-132.

Galagan, J.E., C. Nusbaum, A. Roy et al. 2002. The genome of $M$. acetivorans reveals extensive metabolic and physiological diversity. Genome Res. 12:532-542.

Hallam, S.J., P.R. Girguis, C.M. Preston, P.M. Richardson and E.F. DeLong. 2003. Identification of methyl coenzyme M reductase A $(m c r A)$ genes associated with methane-oxidizing archaea. Appl. Environ. Microbiol. 69:5483-5491.

Hallam, S.J., N. Putnam, C.M. Preston, J.C. Detter, D. Rokhsar, P.M. Richardson and E.F. DeLong. 2004. Reverse methanogenesis: testing the hypothesis with environmental genomics. Science 305: 1457-1462.

Harder, J. 1997. Anaerobic methane oxidation by bacteria employing ${ }^{14} \mathrm{C}$-methane uncontaminated with ${ }^{14} \mathrm{C}$-carbon monoxide. Mar. Geol. 137:13-23.

Hinrichs, K.U., J.M. Hayes, S.P. Sylva, P.G. Brewer and E.F. DeLong. 1999. Methane-consuming archaebacteria in marine sediments. Nature 398:802-805.

Hinrichs, K.U., R.E. Summons, V. Orphan, S.P. Sylva and J.M. Hayes. 2000. Molecular and isotopic analysis of anaerobic methane-oxidizing communities in marine sediments. Org. Geochem. 31: $1685-1701$.

Hoehler, T.M. and M.J. Alperin. 1996. Anaerobic methane oxidation by methanogen-sulfate reducer consortium: geochemical evidence and biochemical considerations. In Microbial Growth on $\mathrm{C}_{1}$ Compounds. Eds. M.E. Lidstrom and F.R. Tabita. Kluwer Academic Publishers, Boston, pp 326-333.

Hoehler, T.M., M.J. Alperin, D.B. Albert and C.S. Martens. 1994. Field and laboratory studies of methane oxidation in an anoxic marine sediment: evidence for a methanogen-sulfate reducer consortium. Global Biogeochem. Cycles 8:451-463.

Klenk, H.P., R.A. Clayton, J.F. Tomb et al. 1998. The complete genome sequence of the hyperthermophilic, sulphate-reducing archaeon Archaeoglobus fulgidus. Nature 394:364-370.

Krüger, M., S. Shima, R. Thauer and F. Widdel. 2003a. Occurrence and activity of methanogenic enzymes in environmental samples dominated by ANME- 1 archaea and their potential role in the anaerobic oxidation of methane. Am. Soc. Microbiol. 103rd General Meeting Abstracts, Washington, DC, p 414.
Krüger, M., A. Meyerdieks, F.O. Glöckner et al. 2003b. A conspicuous nickel protein in microbial mats that oxidize methane anaerobically. Nature 426:878-881.

Michaelis, W., R. Seifert, K. Nauhaus et al. 2002. Microbial reefs in the Black Sea fueled by anaerobic oxidation of methane. Science 297:1013-1015.

Nauhaus, K., A. Boetius, M. Krüger and F. Widdel. 2002. In vitro demonstration of anaerobic oxidation of methane coupled to sulphate reduction in sediment from a marine gas hydrate area. Environ. Microbiol. 4:296-305.

Orphan, V.J., K.U. Hinrichs, W. Ussler, C.K. Paull, L.T. Taylor, S.P. Sylva, J.M. Hayes and E.F. DeLong. 2001a. Comparative analysis of methane-oxidizing archaea and sulfate-reducing bacteria in anoxic marine sediments. Appl. Environ. Microbiol. 67:1922-1934.

Orphan, V.J., C.H. House, K.U. Hinrichs, K.D. McKeegan and E.F. DeLong. 2001b. Methane-consuming archaea revealed by directly coupled isotopic and phylogenetic analysis. Science 293: 484-487.

Orphan, V.J., C.H. House, K.U. Hinrichs, K.D. McKeegan and E.F. DeLong. 2002. Multiple archaeal groups mediate methane oxidation in anoxic cold seep sediments. Proc. Natl. Acad. Sci. USA 99:7663-7668.

Pancost, R.D., J.S. Sinninghe Damsté, S. de Lint, M.J.E.C. van der Maarel, J.C. Gottschal and the Medinaut Shipboard Scientific Party. 2000. Biomarker evidence for widespread anaerobic methane oxidation in Mediterranean sediments by a consortium of methanogenic archaea and bacteria. Appl. Environ. Microbiol. 66: $1126-1132$.

Stetter, K.O., G. Lauerer, M. Thomm and A. Neuner. 1987. Isolation of extremely thermophilic sulfate reducers: evidence for a novel branch of archaebacteria. Science 236:822-824.

Stetter, K.O., R. Huber, E. Blöchl, M. Kurr, R.D. Eden, M. Fielder, H. Cash and I. Vance. 1993. Hyperthermophilic archaea are thriving in deep North Sea and Alaska oil reservoirs. Nature 365: 743-745.

Valentine, D.L. and W.S. Reeburgh. 2000. New perspectives on anaerobic methane oxidation. Environ. Microbiol. 2:477-484.

Valentine, D.L., D.C. Blanton and W.S. Reeburgh. 2000. Hydrogen production by methanogens under low-hydrogen conditions. Arch. Microbiol. 174:415-421.

Wolin, E.A., M.J. Wolin and R.S. Wolfe. 1963. Formation of methane by bacterial extracts. J. Biol. Chem. 238:2882-2886.

Zehnder, A.J.B. and T.D. Brock. 1979. Methane formation and methane oxidation by methanogenic bacteria. J. Bacteriol. 137:420-432.

Zehnder, A.J.B. and T.D. Brock. 1980. Anaerobic methane oxidation: occurrence and ecology. Appl. Environ. Microbiol. 39:194-204. 

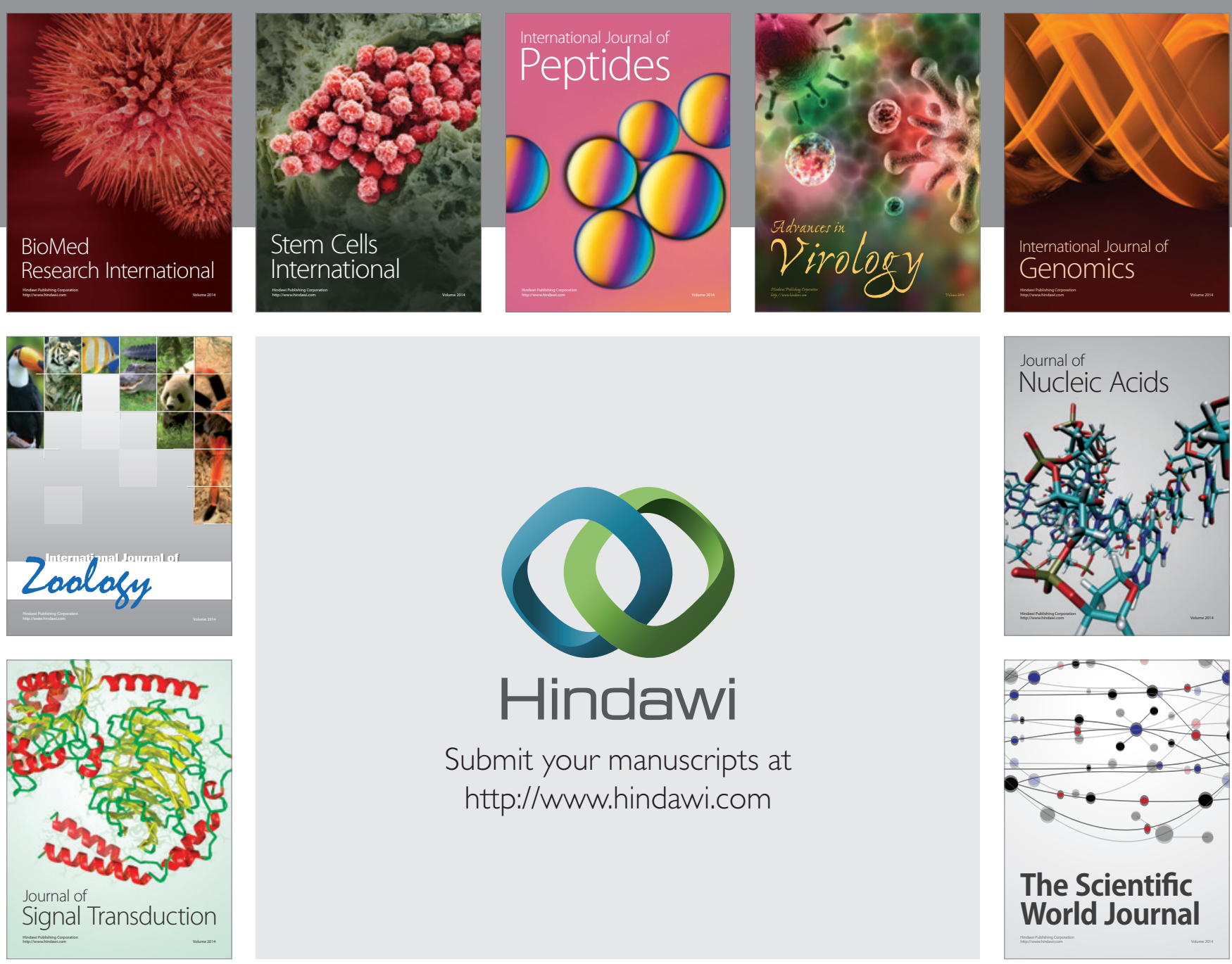

Submit your manuscripts at

http://www.hindawi.com
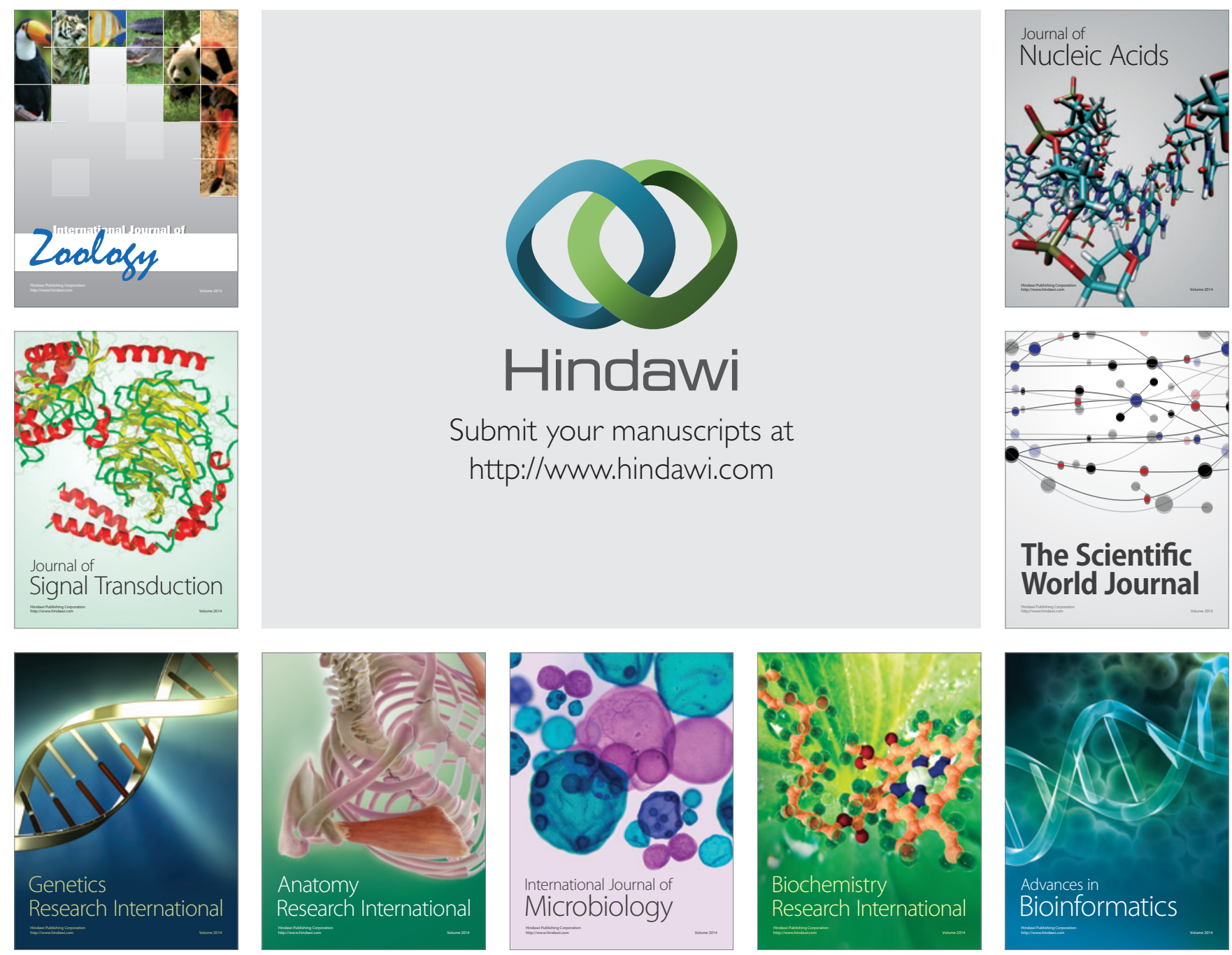

The Scientific World Journal
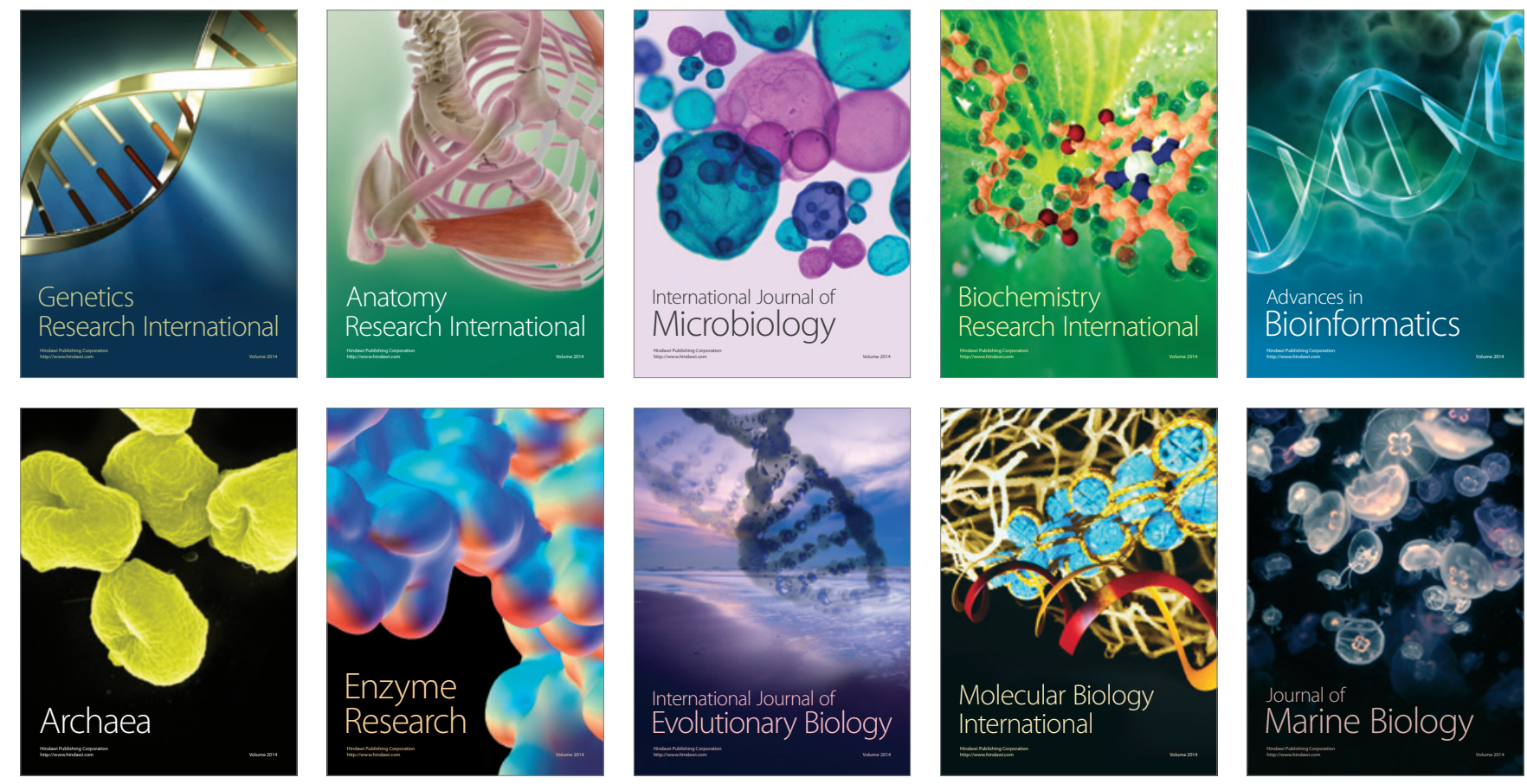